\title{
Liquid-solid interaction at nanoscale and its application in vegetal biology
}

\author{
Henri Gouin \\ C.N.R.S. U.M.R. $6181 \&$ University of Aix-Marseille, \\ Case 322, Av. Escadrille Normandie-Niemen, 13397 Marseille Cedex 20 France
}

\begin{abstract}
+ The water ascent in tall trees is subject to controversy: the vegetal biologists debate on the validity of the cohesion-tension theory which considers strong negative pressures in microtubes of xylem carrying the crude sap. This article aims to point out that liquids are submitted at the walls to intermolecular forces inferring density gradients making heterogeneous liquid layers and therefore disqualifying the NavierStokes equations for nanofilms. The crude sap motion takes the disjoining pressure gradient into account and the sap flow dramatically increases such that the watering of nanolayers may be analogous to a microscopic flow. Application to microtubes of xylem avoids the problem of cavitation and enables us to understand why the ascent of sap is possible for very high trees.
\end{abstract}

Key words: nanofilms; disjoining pressure; cohesion-tension theory; interface motions; Navier length; ascent of sap.

\section{Introduction}

The model we develop makes it possible to investigate the behavior of the fluids in the nanofilms, and its applications extend to life sciences. A particularly interesting example concerns vegetable biology: the rise and the motion of sap in the highest trees focus many polemics and debates between biologists. Many of them regard the approach known as the cohesion-tension theory (CTT) proposed at the end of the nineteenth century by Dixon and Joly as the only valid one [1].

Email address: henri.gouin@univ-cezanne.fr, Telephone: +33 491288 407, Fax: +33 491288776 (Henri Gouin). 
As an obvious fact, Flindt reports huge trees as eucalyptus and giant sequoias of more than 130 meters [2], but the biophysical determination of maximum size to which trees can grow is not well understood and calculated. The main problem with the understanding of water transport is why the sap is able to irrigate up very high levels in tall trees.

The crude sap contains diluted salts but its physical properties are roughly comparable with the water ones. Hydrodynamics, capillarity and osmotic pressure create a ascent of sap of only few tens of meters [3]. To explain the sap ascent phenomenon, Dixon and Joly proposed a cohesion-tension model, followed by a quantitative attempt [4]: liquids are assumed to be subjected to tensions generating negative pressures compensating gravity effects.

As pointed out in [5], a turning-point in the confidence of the opponents to the cohension-tension theory for the sap ascent was the experiment which demonstrated that tall trees survive by overlapping double saw-cuts made through the cross-sectional area of the trunk to sever all xylem elements [6]. This result confirmed by several authors does not seem in agreement with the possibility of strong negative pressures in microtubes $[7,8]$. Using a xylem pressure probe, the apparatus does not measure any water tension in many circumstances: xylem tension exceeding 0.6 Mpa seems not to be observed and in normal state most vessels may be embolized at a level corresponding about sixty meter height. Moreover, gas-vapor transportation in xylem tubes seems to appear at the top of high trees ([5] and its references herein).

As comments and questions, M.H. Zimmerman wrote in 1983 [3]:

"We don't yet fully understand all aspects of xylem-water supply to leaves and have here a wide-open filed of potential very interesting future research. The heartwood is referred to as a wet wood. It may contain liquid under positive pressure while in the sapwood the transpiration stream moves along a gradient of negative pressures. Why is the water of the central wet core not drawn into the sapwood? Free water, i.e. water in tracheids, decreases in successively older layers of wood as the number of embolized tracheids increases. The heartwood is relatively dry i.e. most tracheids are embolized. It is rather ironic that a wound in the wet wood area, which bleeds liquid for a long period of time, thus appears to have the transpiration stream as a source of water, in spite of the fact that the pressure of the transpiration stream is negative most of the time! It should be quite clear by now that a drop in xylem pressure below a critical level causes cavitations and normally puts the xylem out of function permanently. The cause of such a pressure drop can be either a failing of water to the xylem by the roots, or excessive demand by transpiration."

Many proponents of the $C T T$ wrote a letter [9] to protest against the recent review [5]. They said that "the CTT is widely supported by biological scientists as the only theory consistent with the preponderance of data on water transport of plant".

Nonetheless, the problem of possible cavitation in trees remains. Such liquids are strongly metastable and can generate cavitations causing embolisms in xylem tubes made of dead cells [10]. For example, it is interesting to note that 
in xylem tube - where diameters range between 50 and $400 \mu m$ - the crude sap has a surface tension $\gamma_{l v}$ lower than the surface tension of pure water which is $72 \mathrm{cgs}$ at $20^{\circ}$ Celsius. If we consider a microscopic gas-vapor bubble inside the crude sap with diameter $2 R$, the difference between the gas-vapor pressure $P_{\text {vapor }}$ and the liquid sap pressure $P_{\text {liquid }}$ can be expressed by the Laplace formula: $P_{\text {vapor }}-P_{\text {liquid }}=2 \gamma_{l v} / R$. But $P_{\text {vapor }}$ being positive, unstable bubbles must appear when $R \geq-2 \gamma_{l v} / P_{\text {liquid }}$. For a negative pressure $P_{\text {liquid }} \leq-0.6$ $\mathrm{MPa}$ corresponding to more than sixty meters height, we get $R \geq 0.24 \mu \mathrm{m}$. In such a case, dynamical bubbles spontaneously appear from germs naturally existing in a crude liquid and cavitation makes the tubes embolized. Consequently, without any biological known process it is difficult to be convinced that xylem tubes are not embolized when they are filled enough with sap up to altitude significantly more important than one hundred meters corresponding to the highest trees.

Our understanding of the ascent and the motion of sap in very high trees differs from the CTT: at a higher level than a few tens of meters - corresponding to the pulling of water by capillary and osmotic pressure - we assume that xylem microtubes are embolized. In addition, we also assume that a thin liquid film - with a thickness of a few nanometers $[11,12]$ - wets xylem walls up to the top of the tree. At this scale, long range molecular forces stratify liquids and the ratio between tube diameter and sap film thickness allows us to consider tube walls as plane surfaces.

In Section 2, using the calculations presented in $[13,14]$, we reconsider the analytic expression in density-functional theory for a thin heterogeneous liquid film which takes account of the power-law tail behavior dominant in a thin liquid film in contact with a solid [15]. The effects of the vapor bulk bordering the liquid film are simply expressed with an other density-functional located on a mathematical surface. With such a functional, we obtained the equations of equilibrium, motion and boundary conditions [16] for a thin vertical liquid film wetting a vertical solid wall and we computed the liquid layer thickness as a function of the film level; these previous results can be extended to mixtures of fluid and perfect gas [13]. Then, the so-called disjoining pressure of thin liquid layers yields a natural tool for very thin films [11]. The minimal thickness for which a stable wetting film wets a solid wall is associated with the pancake layer when the film is bordering the dry solid wall and corresponds to the maximal altitude $[11,17,18]$. The normal stress vector acting on the wall remains constant through the liquid layer and corresponds to the gas-vapor bulk pressure which is currently the atmospheric pressure and consequently, no negative pressure appears in the liquid layer. At the top of very high trees, the thickness of the sap layer is of a few number of nanometers. The negative pressure is only present for the liquid bulk in micropores. Numerical calculations associated with physical values for water yield the maximal film altitude for a wood material corresponding to a good order of the height of the tallest trees. 
In Section 3, we consider the flow of sap at high levels. For shallow water, the flows of liquids on solids are mainly represented by using the Navier-Stokes equations associated with adherence conditions at the walls. Recent experiments in nanofluidics seem to prove, also for liquids, that at nanoscales corresponding to sap layers at very high tree levels, the conditions of adherence are disqualified $[19,20]$. With the aim of explaining experimental results, we reconsider the fluids as media whose motions generate slips along the walls; so, we can draw consequences differing from results of classically adopted models as reconsidered in [21]. The new model we are presenting reveals an essential difference between the flows of microfluidics and those of nanofluidics. In the latter, simple laws of scales cannot be only taken anymore into account.

The transpiration in the leaves induces a variation of the sap layer thickness in microtubes. Consequently, the gradient of thickness along microtubes creates a gradient of disjoining pressure which induces driving forces along the layer. For thin layers, the sap flow depending on the variations of the layer thickness can be adapted to each level of leaves following the tree requirement. This is an important understanding why the flow of sap can be non negligible at a level corresponding to the top of the tallest trees. Moreover, we notice that the stability criterium of the flow issued from the equation of motion fits with the results of Derjaguin's school [11].

\section{$2 \quad$ A study of inhomogeneous fluids near a solid wall}

In this section, we recall the main results presented in $[13,14]$. Thanks to these results, in Section 3, we shall consider sap layers of the highest trees with a thickness of some nanometers only.

The density-functional of an inhomogeneous fluid in a very thin isothermal layer domain $(O)$ of wall boundary $(S)$ and liquid-vapor interface $(\Sigma)$ was chosen in the form:

$$
F=\iiint_{(O)} \varepsilon d v+\iint_{(S)} \phi d s+\iint_{(\Sigma)} \psi d s
$$

- The first integral is associated with a square-gradient approximation when we introduce a specific free energy of the fluid at a given temperature $T$ as a function of density $\rho$ and $\beta=(\operatorname{grad} \rho)^{2}$ such as $[22,23]$ :

$$
\rho \varepsilon=\rho \alpha(\rho)+\frac{\lambda}{2}(\operatorname{grad} \rho)^{2},
$$

where term $(\lambda / 2)(\operatorname{grad} \rho)^{2}$ is added to the volume free energy $\rho \alpha(\rho)$ of a compressible fluid and scalar $\lambda$ is assumed to be constant at a given temperature [24]. Specific free energy $\alpha$ enables liquid and vapor bulks to be continuously connected and the pressure $P(\rho)=\rho^{2} \alpha_{\rho}^{\prime}(\rho)$ is similar to van der Waals one. 
- For a plane solid wall $(S)$, the solid-liquid surface free energy is in the form $[15,25]$ :

$$
\phi(\rho)=-\gamma_{1} \rho+\frac{1}{2} \gamma_{2} \rho^{2}
$$

Here $\rho$ denotes the fluid density value at surface $(S)$; constants $\gamma_{1}, \gamma_{2}$ and $\lambda$ are generally positive and given by the mean field approximation:

$$
\gamma_{1}=\frac{\pi c_{l s}}{12 \delta^{2} m_{l} m_{s}} \rho_{s o l}, \quad \gamma_{2}=\frac{\pi c_{l l}}{12 \delta^{2} m_{l}^{2}}, \quad \lambda=\frac{2 \pi c_{l l}}{3 \sigma_{l} m_{l}^{2}},
$$

where $c_{l l}$ and $c_{l s}$ are two positive constants associated with Hamaker constants; $\sigma_{l}$ and $\sigma_{s}$ denoting fluid and solid molecular diameters, $\delta=\frac{1}{2}\left(\sigma_{l}+\sigma_{s}\right) ; m_{l}$, $m_{s}$ denote masses of fluid and solid molecules; $\rho_{s o l}$ is the solid density.

- For the plane liquid-vapor interface $(\Sigma)$ the surface free energy $\psi$ is reduced to $[13,14]$ :

$$
\psi(\rho)=\frac{\gamma_{4}}{2} \rho^{2},
$$

where $\rho$ is the density of the liquid bounding the interface and $\gamma_{4}$ is associated with the interfacial thickness of the order of the fluid molecular diameter $\left(\gamma_{4} \simeq \gamma_{2}\right)$

In case of equilibrium, functional (1) is stationary and yields the equation of equilibrium and boundary conditions $[16,26]$.

\subsection{Equation of equilibrium}

The equation of equilibrium is $[16,27]$ :

$$
\operatorname{div} \boldsymbol{\sigma}+\rho g \mathbf{i}=0
$$

where $\boldsymbol{\sigma}=-\left(\rho^{2} \varepsilon_{\rho}^{\prime}-\rho \operatorname{div}(\lambda \operatorname{grad} \rho)\right) \mathbf{1}-\lambda \operatorname{grad} \rho \otimes \operatorname{grad} \rho, g$ is the acceleration of gravity and $\mathbf{i}$, of coordinate $x$, is the downward direction. Let us consider an isothermal vertical film of liquid; then in orthogonal system, the coordinate $z$ being external and normal to the flat vertical solid wall, spatial density derivatives are negligible except in direction of $z$. In the complete liquid-vapor layer (we call interlayer) and along direction z, Eq. (4) yields a constant value at each level $x$ :

$$
P(\rho)+\frac{\lambda}{2}\left(\frac{d \rho}{d z}\right)^{2}-\lambda \rho \frac{d^{2} \rho}{d z^{2}}=P_{v_{b_{x}}}
$$

where $P_{v_{b_{x}}}=P\left(\rho_{v_{b_{x}}}\right)$ denotes the pressure in the vapor bulk of density $\rho_{v_{b_{x}}}$ bounding the liquid layer at level $x$. In the fluid, Eq. (4) can be written [27]:

$$
\operatorname{grad}\left(\mu_{o}-\lambda \Delta \rho-g x\right)=0
$$


where $\mu_{o}$ is the chemical potential (at temperature $T$ ), chosen null in the liquid and vapor bulks of phase equilibrium densities $\rho_{l}$ and $\rho_{v}$, respectively; $\Delta$ denotes the Laplacian. Thanks to Eq. (5), we obtain in all the fluid and not only in the interlayer:

$$
\mu_{o}(\rho)-\lambda \Delta \rho-g x=\mu_{o}\left(\rho_{b}\right)
$$

where $\mu_{o}\left(\rho_{b}\right)$ is the chemical potential value of a liquid mother bulk of density $\rho_{b}$ such that $\mu_{o}\left(\rho_{b}\right)=\mu_{o}\left(\rho_{v_{b}}\right)$, where $\rho_{v_{b}}$ is the density of the vapor bulk bounding the layer at level $x=0$ [11]. Equation (6) is valid in the interlayer and yields the equation of density profile:

$$
\lambda \frac{d^{2} \rho}{d z^{2}}=\mu_{b_{x}}(\rho), \quad \text { with } \quad \mu_{b_{x}}(\rho)=\mu_{o}(\rho)-\mu_{o}\left(\rho_{b_{x}}\right)
$$

where $\rho_{b_{x}}$ is the liquid mother bulk density at level $x$.

\subsection{The disjoining pressure for vertical liquid films}

The disjoining pressure at level $x$ can be written as [11]:

$$
\Pi=P_{v_{b_{x}}}-P_{b_{x}}
$$

where $P_{b_{x}}=P\left(\rho_{b_{x}}\right)$. At a given temperature $T$, $\Pi$ is a function of $\rho_{b_{x}}$. The reference chemical potential linearized near $\rho_{l}$ is $\mu_{o}(\rho)=\left(c_{l}^{2} / \rho_{l}\right)\left(\rho-\rho_{l}\right)$ where $c_{l}$ is the isothermal sound velocity in liquid bulk $\rho_{l}$ at temperature $T$ [28]. In the liquid part of the liquid-vapor film, Eq. (7) writes:

$$
\lambda \frac{d^{2} \rho}{d z^{2}}=\frac{c_{l}^{2}}{\rho_{l}}\left(\rho-\rho_{b}\right)-g x \equiv \frac{c_{l}^{2}}{\rho_{l}}\left(\rho-\rho_{b_{x}}\right) \quad \text { with } \rho_{b_{x}}=\rho_{b}+\frac{\rho_{l}}{c_{l}^{2}} g x .
$$

At level $x=0$, the liquid mother bulk density is closely equal to $\rho_{l}$ and because of Rel. (8), $\Pi$ can be considered as a function of $x$ [13]:

$$
\Pi(x)=-\rho_{l} g x\left(1+\frac{g x}{2 c_{l}^{2}}\right) .
$$

Now, we consider a film of thickness $h_{x}$ at level $x$; the density profile in the liquid part of the liquid-vapor film is solution of the system:

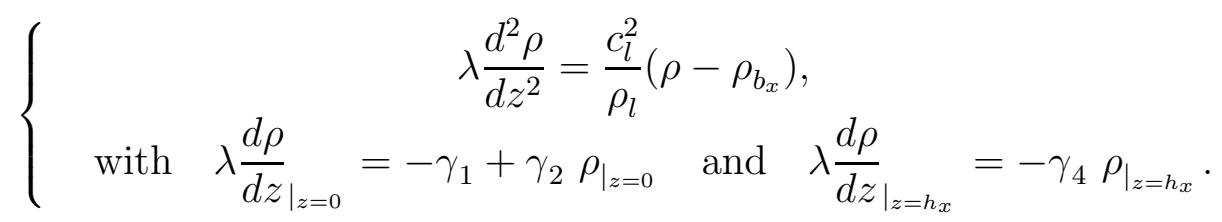


Let quantities $\tau, d$ and $\gamma_{3}$ be defined as:

$$
\tau \equiv \frac{1}{d}=\frac{c_{l}}{\sqrt{\lambda \rho_{l}}} \quad \text { and } \quad \gamma_{3} \equiv \lambda \tau
$$

such that $d$ is a reference length. Due to the fact that $\rho_{b_{x}} \simeq \rho_{b} \simeq \rho_{l}[11]$, the disjoining pressure reduces to [14]:

$$
\begin{aligned}
\Pi\left(h_{x}\right)= & \frac{2 c_{l}^{2}}{\rho_{l}}\left[\left(\gamma_{1}-\gamma_{2} \rho_{l}\right)\left(\gamma_{3}+\gamma_{4}\right) e^{h_{x} \tau}+\left(\gamma_{2}-\gamma_{3}\right) \gamma_{4} \rho_{l}\right] \times \\
& \frac{\left[\left(\gamma_{2}+\gamma_{3}\right) \gamma_{4} \rho_{l}-\left(\gamma_{1}-\gamma_{2} \rho_{l}\right)\left(\gamma_{3}-\gamma_{4}\right) e^{-h_{x} \tau}\right]}{\left[\left(\gamma_{2}+\gamma_{3}\right)\left(\gamma_{3}+\gamma_{4}\right) e^{h_{x} \tau}+\left(\gamma_{3}-\gamma_{4}\right)\left(\gamma_{2}-\gamma_{3}\right) e^{-h_{x} \tau}\right]^{2}} .
\end{aligned}
$$

The disjoining pressure of the mixture of liquid and perfect gas is the same than for a single van der Waals fluid and calculations and results are identical to those previously obtained [13].

\subsection{Water wetting a vertical plane wall of xylem}

Our aim is to point out an example such that previous results provide a value of maximum height for a vertical water film wetting a plane wall of xylem and to estimate the sap layer thickness at this altitude.

As proved by Derjaguin et al in [11], (Chapter 2), the Gibbs free energy per unit area $G$ can be expressed as a function of $h_{x}$ :

$$
G\left(h_{x}\right)=\int_{h_{x}}^{+\infty} \Pi(h) d h
$$

where $h_{x}=0$ is associated with the dry wall in contact with the vapor bulk and $h_{x}=+\infty$ is associated with a wall in contact with the liquid bulk. The spreading coefficient is $S=\gamma_{S V}-\gamma_{S L}-\gamma_{L V}$, where $\gamma_{S V}, \gamma_{S L}, \gamma_{L V}$ are the solid-vapor, solid-liquid and liquid-vapor free energies per unit area of interfaces, respectively. The energy of the liquid layer per unit area can be written $E=\gamma_{S L}+\gamma_{L V}+G\left(h_{x}\right)$.

The coexistence of two film segments with different thicknesses is a phenomenon interpreted with the equality of chemical potentials and the equality surface tensions of the two films. A spectacular case corresponds to the coexistence of a liquid film of thickness $h_{p}$ and a dry solid wall associated with $h_{x}=0$. The film is the so-called pancake layer corresponding to the condition $[11,17]$ :

$$
G(0)=G\left(h_{p}\right)+h_{p} \Pi\left(h_{p}\right) .
$$

Liquid films of thickness $h_{x}>h_{p}$ are stable and liquid films of thickness $h_{x}<h_{p}$ are metastable or unstable. For a few nanometer range, the film 
thickness is not exactly $h_{x}$; we must add the thickness estimated at $2 \sigma_{l}$ of the liquid part of the liquid-vapor interface bordering the liquid layer and the film thickness is $e_{x} \approx h_{x}+2 \sigma_{l}[23,24]$.

When $h_{x}=0$ (corresponding to the dry wall), the value of $G$ is the spreading coefficient $S$. Point $P$ associated with the pancake layer is observed on the curve to be closely an inflexion point of graph $\Pi\left(h_{x}\right)$ [13]. To obtain the pancake thickness corresponding to the smallest film thickness, we draw the graphs of $\Pi\left(h_{x}\right)$ and $G\left(h_{x}\right)$ when $h_{x} \in\left[(1 / 2) \sigma_{l}, \ell\right]$, where $\ell$ is a distance of few tens of Amgström.

For the numerical calculations, we considered water at $T=20^{\circ}$ Celsius wetting a wall in xylem. The experimental estimates of coefficients are obtained in c.g.s. units $[12,14,29]$ :

$\rho_{l}=0.998, c_{l}=1.478 \times 10^{5}, c_{l l}=1.4 \times 10^{-58}, \sigma_{l}=2.8 \times 10^{-8}$ (2.8 ångströms), $m_{l}=2.99 \times 10^{-23}$. From Rel. (3), we deduce $\lambda=1.17 \times 10^{-5}, \gamma_{2}=\gamma_{4}=54.2$. From Rel. (10), we get $\gamma_{3}=506, d=2.31 \times 10^{-8}$.

We consider a material such that the Young angle between the liquid-vapor interface and the solid material surface is $\theta \approx 50^{\circ}$. This Young angle is an arithmetic average of Young angles for water wetting different xylem walls [30]. The coefficient $c_{l s}$ is obviously not given in the physical tables and $\gamma_{1}$ cannot be obtain from Rel. (3); due to Rel. (2), we immediately get the unknown coefficient $\gamma_{1}$ in Rel. $(11): \gamma_{1} \approx 75$.

In Fig. 1 - left graph, we present the disjoining pressure graph $\Pi\left(h_{x}\right)$. The physical part of disjoining pressure graph corresponding to $\partial \Pi / \partial h_{x}<0$ is a plain line and is associated with thickness liquid layer of several molecules. The dashed line has no real existence.

In Fig. 1 - right graph, we present the free energy graph $G\left(h_{x}\right)$. Due to $h_{x}>(1 / 2) \sigma_{l}$, it is not possible to obtain the limit point $W$ corresponding to the dry wall. This point is obtained by an interpolation associated with the concave part of the $G$-curve. Point $P$ follows from the drawing of the tangent line issued from $W$ to the $G$-curve. The limit of the film thickness is associated to the pancake thickness $e_{p} \approx h_{p}+2 \sigma_{l}$ when the liquid film coexists with the dry wall. The reference length $d$ is of the same order than $\sigma_{l}, \sigma_{s}$ and $\delta$ and is a good length order for very thin films. The total pancake thickness $e_{p}$ is of one nanometer order corresponding to a good thickness value for a highenergy surface [18]. We deduce $S \approx 40$ cgs. However, crude sap is not pure water. Its liquid-vapor surface tension has a lower value than surface tension of pure water $\left(72 \mathrm{cgs}\right.$ at $\left.20^{\circ} \mathrm{C}\right)$ and it is possible to obtain the same spreading coefficients with less energetic surfaces.

When $|x|$ is of some hundred meters, Eq. (9) yields $\Pi(x) \simeq-\rho_{l} g x$.

The maximum of altitude $\left|x_{M}\right|$ corresponds to the pancake layer. To this altitude, we add 20 meters corresponding to the ascent of sap due to capillarity and osmotic pressure and we obtain a film height of 140 meters which is of the same order than the topmost trees. We also note the important result: in 
the trees, the thickness of the layer is of some nanometers at high level.
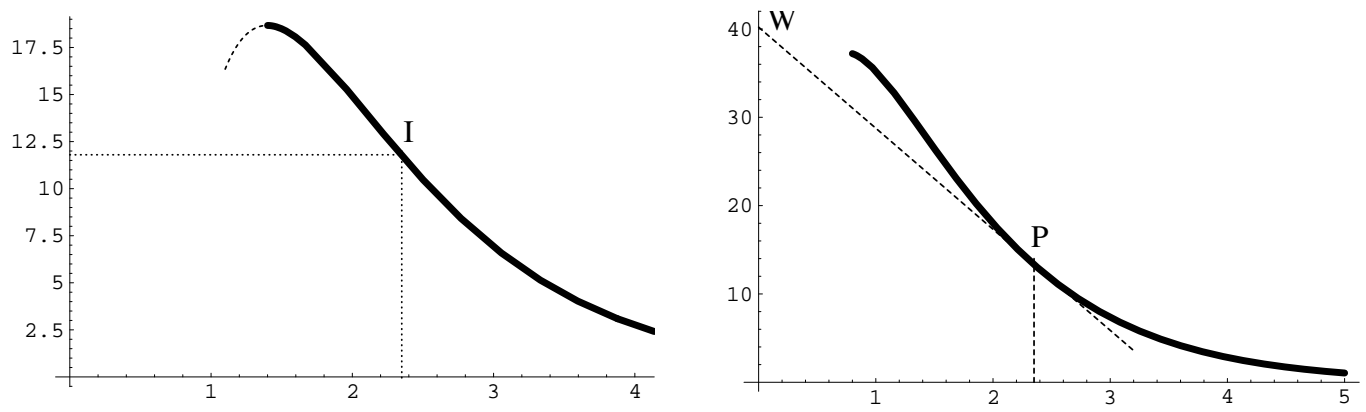

Fig. 1. Left graph: $\Pi\left(h_{x}\right)$-graph. The unit of $x$-axis is $d=2.31 \times 10^{-8} \mathrm{~cm}$; the unit of $y$-axis is one atmosphere. Right graph: $G\left(h_{x}\right)$-graph. The unit of $x$-axis is $d=2.31 \times 10^{-8} \mathrm{~cm}$; the unit of $y$-axis is one cgs unit of surface tension.

\section{Dynamics of liquid nanofilms}

The idea of xylem tubes completely filled with sap induces that the flow of liquid along vessels can be compared with a flow through capillaries [3]. The flow rate through capillary tubes is proportional to the applied pressure gradient and the hydraulic conductivity. Parabolic flow causes the flow rate to be proportional to the fourth power of capillary radius [31]. One of the most important design requirements is that vapor blockage does not happen in the stem. Consequently, to be efficient for the transport of sap, the xylem tube radius must be as larger as possible, which is not the case.

The dynamics of such liquid nanofilms is always studied in isothermal case. Our model of sap nanolayer implies different consequences:

- The classical model by Navier-Stokes is unable to describe fluid motions in very thin films,

- The notion of surface free energy of a sharp interface separating gas and liquid layer must be reconsidered,

- The equation of fluid motions along the nanofilm is obtained by adding the forces of viscosity to the conservative forces,

- The evolution equation of the film thickness takes account of the variation of the disjoining pressure along the layer.

At equilibrium, the different fluid quantities are $\rho_{b_{x}}, \rho_{v_{b_{x}}}, P_{b_{x}}, P_{v_{b_{x}}}, h_{x} \ldots$ For the motion, the corresponding quantities are denoted by $\rho_{b_{x}}^{*}, \rho_{v_{b_{x}}}^{*}, P_{b_{x}}^{*}, P_{v_{b_{x}}}^{*}, h_{x}^{*} \ldots$ When the liquid layer thickness is small with respect to transverse dimensions of the wall, it is possible to simplify the Navier-Stokes equation which governs the flow of classical viscous fluids.

When $h \ll L$, where $L$ is the wall transversal characteristic size,

i) The velocity component along the wall is large with respect to the normal velocity component which can be neglected, 
ii) The velocity vector mainly varies along the direction orthogonal to the wall and it is possible to neglect velocity derivatives with respect to coordinates along the wall compared to the normal derivative,

iii) The pressure is constant in the direction normal to the wall. It is possible to neglect the inertial term when $R e \ll L / h$, where $R e$ is the Reynolds number of the flow.

Equation of hydrodynamics is not valid in a liquid nanolayer because the fluid is heterogeneous and the liquid stress tensor is not anymore scalar. However, it is possible to adapt the results obtained for viscous flows to motions in liquid nanolayers: due to $\epsilon=h / L \ll 1$, we are also in the case of long wave approximation.

We denote the velocity by $\mathbf{V}=(u, v, w)$ where $(u, v)$ are the tangential components. Due to the fact that $e=\sup (|w / u|,|w / v|) \ll 1$, we also are in the case of approximation of lubrication. The main parts of terms associated with second derivatives of liquid velocity components correspond to $\partial^{2} u / \partial z^{2}$ and $\partial^{2} v / \partial z^{2}$. The density is constant along each stream line $(\dot{\rho}=0 \Longleftrightarrow \operatorname{div} \mathbf{V}=0)$ and isodensity surfaces contain the trajectories. Then, $\partial u / \partial x, \partial v / \partial y$ and $\partial w / \partial z$ have the same order of magnitude and $\epsilon \sim e$.

As in [24], we assume that the kinematic viscosity coefficient $\nu=\kappa / \rho$ only depends on the temperature. In motion equation, the viscosity term is

$$
(1 / \rho) \operatorname{div} \boldsymbol{\sigma}_{v}=2 \nu[\operatorname{div} \boldsymbol{D}+\boldsymbol{D} \operatorname{grad}\{\operatorname{Ln}(2 \kappa)\}],
$$

where $\boldsymbol{D}$ is the velocity deformation tensor and $\boldsymbol{D} \operatorname{grad}\{\operatorname{Ln}(2 \kappa)\}$ is negligible with respect to $\operatorname{div} \boldsymbol{D}$.

In both lubrication and long wave approximations, the liquid nanolayer motion verifies [27]:

$$
\boldsymbol{\Gamma}+\operatorname{grad}\left[\mu_{o}(\rho)-\lambda \Delta \rho\right]=\nu \Delta \mathbf{V}+g \mathbf{i} \text { with } \Delta \mathbf{V} \simeq\left[\frac{\partial^{2} u}{\partial z^{2}}, \frac{\partial^{2} v}{\partial z^{2}}, 0\right]
$$

This equation corresponds to the equation of equilibrium (4) with addition of inertial (acceleration) term $\boldsymbol{\Gamma}$ and viscous term $\nu \Delta \mathbf{V}$.

In approximation of lubrication, the inertial term is neglected:

$$
\operatorname{grad}\left[\mu_{o}(\rho)-\lambda \Delta \rho\right]=\nu \Delta \mathbf{V}+g \mathbf{i}
$$

Equation (12) separates into tangential and normal components to the solid wall.

- The normal component - following $z$ - of Eq. (12) writes in the same form than for equilibrium:

$$
\frac{\partial}{\partial z}\left[\mu_{o}(\rho)-\lambda \Delta \rho\right]=0
$$

and consequently,

$$
\mu_{o}(\rho)-\lambda \Delta \rho=\mu_{o}\left(\rho_{b_{x}}^{*}\right)
$$


where $\rho_{b_{x}}^{*}$ is the dynamical liquid mother bulk density at level $x$ (different from the liquid bulk density $\rho_{l}$ of the plane interface at equilibrium and also from $\rho_{b_{x}}$, liquid mother bulk density at level $x$ at equilibrium).

To each density $\rho_{b_{x}}^{*}$ is associated a liquid nanolayer thickness $h_{x}^{*}$. We can write $\mu_{o}\left(\rho_{b}\right)-\lambda \Delta \rho=\eta\left(h_{x}^{*}\right)$, where $\eta$ is such that $\eta\left(h_{x}^{*}\right)=\mu_{o}\left(\rho_{b_{x}}^{*}\right)$.

- For one-dimensional motions colinear to the solid wall (direction $\mathbf{i}$ and velocity $u \mathbf{i}$ ), by taking account of Eq. (13), the tangential component of Eq. (12) yields:

$$
\mathbf{i} \cdot \operatorname{grad} \mu_{o}\left(\rho_{b_{x}}^{*}\right)=\nu \frac{\partial^{2} u}{\partial z^{2}}+g
$$

which is equivalent to:

$$
\frac{\partial \mu_{o}\left(\rho_{b_{x}}^{*}\right)}{\partial \rho_{b_{x}}^{*}} \frac{\partial \rho_{b_{x}}^{*}}{\partial x}=\nu \frac{\partial^{2} u}{\partial z^{2}}+g .
$$

For the most practical situations, simple fluids slip on a solid wall only at a molecular level [32] and consequently, the kinematic condition at solid walls is the adherence condition $(z=0 \Rightarrow u=0)$. Nevertheless, with water flowing on thin hydrophobic nanolayers, there are some qualitative observations for slippage. With water flowing in thin, hydrophobic capillaries, there are also some early qualitative evidences for slippage [33]. De Gennes said: "the results are unexpected and stimulating and led us to think about unusual processes which could take place near a wall. They are connected with the thickness $h$ of the film when $h$ of an order of the mean free path" [19].

Recent papers in nonequilibrium molecular dynamics simulations of three dimensional micro-Poiseuille flows in Knudsen regime reconsider microchannels: the influence of gravity force, surface roughness, surface wetting condition and wall density are investigated. The results point out that the no-slip condition can be observed for Knudsen flow when the surface is rough. The roughness is a dominant parameter as far as the slip of fluid is concerned. The surface wetting condition substantially influences the velocity profiles [20]. In fluid/wall slippage, the condition at solid wall writes:

$$
u=L_{s} \frac{\partial u}{\partial z} \quad \text { at } z=0
$$

where $L_{s}$ is the so-called Navier length. The Navier length is expected to be independent of $h$ and may be as large as a few microns [20].

At the liquid-vapor interface, we also assume that vapor viscosity stress is negligible; from the continuity of fluid tangential stress through a liquid-vapor interface, we get:

$$
\frac{\partial u}{\partial z}=0 \quad \text { at } z=h_{x}^{*}
$$


Consequently, Eq. (14) implies:

$$
\nu u=\left(\frac{\partial \mu_{o}\left(\rho_{b_{x}}^{*}\right)}{\partial \rho_{b_{x}}^{*}} \frac{\partial \rho_{b_{x}}^{*}}{\partial x}-g\right)\left(\frac{1}{2} z^{2}-h_{x}^{*} z-L_{s} h_{x}^{*}\right) .
$$

The mean spatial velocity $\bar{u}$ of the liquid in the nanolayer at level $x$ is:

$$
\bar{u}=\frac{1}{h_{x}^{*}} \int_{o}^{h_{x}^{*}} u d z .
$$

Previous computations yield:

$$
\nu \overline{\mathbf{u}}=-h_{x}^{*}\left(\frac{h_{x}^{*}}{3}+L_{s}\right)\left[\operatorname{grad} \mu_{o}\left(\rho_{b_{x}}^{*}\right)-g \mathbf{i}\right] \quad \text { with } \quad \overline{\mathbf{u}}=\bar{u} \mathbf{i} .
$$

Let us note that:

$$
\frac{\partial \mu_{o}\left(\rho_{b_{x}}^{*}\right)}{\partial x}=\frac{\partial \mu_{o}\left(\rho_{b_{x}}^{*}\right)}{\partial \rho_{b_{x}}^{*}} \frac{\partial \rho_{b_{x}}^{*}}{\partial h_{x}^{*}} \frac{\partial h_{x}^{*}}{\partial x} \equiv \frac{1}{\rho_{b_{x}}^{*}} \frac{\partial P\left(\rho_{b_{x}}^{*}\right)}{\partial \rho_{x}^{*}} \frac{\partial \rho_{b_{x}}^{*}}{\partial h_{x}^{*}} \frac{\partial h_{x}^{*}}{\partial x} .
$$

Due to the fact the vapor bulk pressure $P_{v_{b_{x}}}^{*}$ is constant along the xylem tube, by using relation $\Pi\left(h_{x}^{*}\right)=P_{v_{b_{x}}}^{*}-P_{b_{x}}^{*}$, we get along the flow motion:

$$
\frac{\partial \mu_{o}\left(\rho_{b_{x}}^{*}\right)}{\partial x}=-\frac{1}{\rho_{b_{x}}^{*}} \frac{\partial \Pi\left(h_{x}^{*}\right)}{\partial h_{x}^{*}} \frac{\partial h_{x}^{*}}{\partial x}
$$

and consequently,

$$
\chi_{b_{x}}^{*} \overline{\mathbf{u}}=h_{x}^{*}\left(\frac{h_{x}^{*}}{3}+L_{s}\right)\left[\operatorname{grad} \Pi\left(h_{x}^{*}\right)+g \mathbf{i}\right],
$$

where $\chi_{b_{x}}^{*}=\rho_{b_{x}}^{*} \nu$ is the liquid kinetic viscosity. Consequently, the mean liquid velocity is driven by the variation of the disjoining pressure along the solid wall and the film thickness. Equation (15) differs from the classical film hydrodynamic one. Indeed, for a classical thin liquid film, the Darcy law is $\overline{\mathbf{u}}=-K(h) \operatorname{grad} p$, where $p$ is the liquid pressure and $K(h)$ is the permeability coefficient. In Eq. (15), the sign is opposite and the liquid pressure is replaced by the disjoining pressure. We note that $\chi_{b_{x}}^{*} \simeq \chi$, where $\chi$ is the liquid kinetic viscosity in the liquid bulk at phase equilibrium. Moreover, when $h_{x}^{*} / L_{s} \ll 1$,

$$
\chi \overline{\mathbf{u}}=h_{x}^{*} L_{s}\left[\operatorname{grad} \Pi\left(h_{x}^{*}\right)+g \mathbf{i}\right],
$$

which is strongly different from the case $L_{s}=0$ corresponding to the adherence condition:

$$
\chi \overline{\mathbf{u}}=\frac{h_{x}^{* 2}}{3}\left[\operatorname{grad} \Pi\left(h_{x}^{*}\right)+g \mathbf{i}\right] .
$$

The mass equation averaged over the liquid depth is:

$$
\frac{\partial}{\partial t}\left(\int_{0}^{h_{x}^{*}} \rho d z\right)+\operatorname{div}\left(\int_{0}^{\mathrm{h}_{\mathrm{x}}^{*}} \rho \mathbf{u d z}\right)=0 .
$$


Since the variation of density is small in the liquid nanolayer, the equation for the free surface is:

$$
\frac{d h_{x}^{*}}{d t}+h_{x}^{*} \operatorname{div} \overline{\mathbf{u}}=0
$$

By replacing (15) into (16) we finally get:

$$
\frac{\partial h_{x}^{*}}{\partial t}+\frac{1}{\chi} \operatorname{div}\left\{h_{x}^{* 2}\left(\frac{h_{x}^{*}}{3}+L_{s}\right)\left[\operatorname{grad} \Pi\left(\mathrm{h}_{\mathrm{x}}^{*}\right)+\mathrm{g} \mathbf{i}\right]\right\}=0 .
$$

It is easy to verify that Eq. (17) is a non-linear parabolic equation.

If $\partial \Pi\left(h_{x}^{*}\right) / \partial h_{x}^{*}<0$ the flow is stable. This result is in accordance with the static criterium of stability for thin liquid layers [11].

When $L_{s} \neq 0$ we notice the flow is multiplied by the factor $1+3 L_{s} / h_{x}^{*}$. For example, when $h_{x}^{*}=3 \mathrm{~nm}$ and $L_{s}=100 \mathrm{~nm}$ which is a Navier length of small magnitude with respect with experiments, the multiplier factor is $10^{2}$; when $L_{s}$ is $7 \mu \mathrm{m}$ as considered in [20], the multiplier factor is $10^{4}$, which seems possible in nanotube observations [30].

Equation (15) yields the flow rate per unit of length of xylem tubes. We may remark that Eq. (15) is mainly valid at the top of highest trees where the xylem tube network is strongly ramified.

A main difference between Poiseuille flow and motion in a thin film is the versatility of the liquid layer flow with respect to Poiseuille'. An hydraulic Poiseuille flow is very rigid due to the liquid incompressibility, the pressure effects are fully propagated in all the tube. For a thin layer flow, the flow rate can increase or decrease due to the spatial derivative of $h_{x}^{*}$ and strongly depends on the locally defined disjoining pressure. Trees can adapt the disjoining pressure effects by opening or closing the stomatic cells with the object of changing the evaporation in its leaves so that the bulk pressure in micropores can be negative and the transport of water can be differently dispatched in the tree parts; this seems an important aspect of the model.

\section{Discussion and conclusions}

Our model is essentially different from the cohesion-tension theory; it allows a new explanation of biofluidics by using methods of the Russian school of Derjaguin and using non-adherence conditions for nanofluid flows at xylem walls. It precisely gives the possible height of the tallest trees. In the very thin layers, we obtain rates of flows strongly larger than those obtained with traditional Navier-Stokes models. The explanation of irrigation of the leaves in the tallest trees supplementary justifies our model of biomimetism.

The motor of the sap motion is induced by the transpiration across micropores located in tree leaves [3]. It is natural to conjecture that the diameters

of xylem tubes must be the result of a competition between evaporation in 
tubes which reduces the flow of sap and the flux of transpiration in micropores inducing the motion strength.

We notice that the negative pressure only appears in the liquid mother bulk. The microlimbs, micropores and stomates have a diameter a little smaller than the bubble size considered in the introduction; consequently, they can be filled without any cavitation by the liquid mother bulk at a suitable negative pressure associated with the height of the tallest trees.

It is interesting to note that if we switch the microtube surfaces to wedge geometry or to corrugated surface, it is much easier to obtain the complete wetting requirement; thus, plants can avoid having very high energy surfaces. Nonetheless, they are still internally wet if crude sap flows pass through wedge shaped corrugated pores; this fact also answers to questions in [3]. The wedge does not have to be perfect on the nanometer scale to significantly enhance the amount of liquid flowing at modest pressures corresponding to nanosized planar films. It is bound to improve on the calculation because it enhances the surface to volume ratio. In such a case, we remark that the wall boundary can always be considered as a plane surface endowed with an average surface energy as in Wenzel's formula [34].

Finally, it will be interesting to confirm our theoretical predictions with additional experimental data.

\section{References}

[1] H.H. Dixon, J. Joly, On the ascent of sap, Phil. Trans. Roy. Soc. London, B 186 (1894) 563-576.

[2] R. Flindt, Amazing Numbers in Biology, Springer, Berlin, 2006.

[3] M.H. Zimmermann, Xylem structure and the ascent of sap, Springer, Berlin, 1983.

[4] T.H. van der Honert, Water transport in plants as a catenary process, Discussions of the Faraday Society 3 (1948) 1105-1113.

[5] U. Zimmermann, H. Schneider, L.H. Wegner, A. Haase, Water ascent in tall trees: does evolution of land plants rely on a highly metastable state? Tansley review, New Phytologist 162 (2004) 575-615.

[6] R.D. Preston, Movement of water in higher plants, in: A. Frey-Wyssling (Ed.), Deformation and Flow in Biological Systems, North Holland Publishing, Amsterdam, 1952, pp. 257-321.

[7] J.F.G. Mackay, P.E. Weatherley, The effects of transverse cuts through the stems of transpiring woody plants on water transport and stress in the leaves, J. of Exp. Botany 24 (1973) 15-28. 
[8] R. Benkert, A. Balling, U. Zimmermann, Direct measurements of the pressure and flow in the xylem vessels of nicotiana tabacum and their dependence on flow resistance and transpiration rate, Botanica Acta 104 (1991) 423-432.

[9] G. Angeles et al., The cohension-tension theory, New Phytologist 163 (2004) 451-452.

[10] M.T. Tyree, The cohesion-tension theory of sap ascent: current contreversies, J. Exp. Botany 48 (1997) 1753-1765.

[11] B.V. Derjaguin, N.V. Chuarev, V.M. Muller, Surfaces Forces, Plenum Press, New York, 1987.

[12] J. Israelachvili, Intermolecular Forces, Academic Press, New York, 1992.

[13] H. Gouin, A new approach for the limit to tree height using a liquid nanolayer model, Continuum Mech. Thermodyn. 20 (2008) 317-329, arXiv:0809.3529.

[14] H. Gouin, Liquid nanofilms. A mechanical model for the disjoining pressure, Int. J. Eng. Sci. 47 (2009) 691-699, arXiv:0904.1809.

[15] H. Gouin, Energy of interaction between solid surfaces and liquids, J. Phys. Chem. B 102 (1998) 1212-1218, arXiv:0801.4481.

[16] H. Gouin, W. Kosiński, Boundary conditions for a capillary fluid in contact with a wall, Arch. Mech. 50 (1998) 907-916, arXiv:0802.1995.

[17] P.G. de Gennes, Wetting: statics and dynamics, Rev. Mod. Phys. 57 (1985) 827-863.

[18] P.G. de Gennes, F. Brochard-Wyart, D. Quéré, Capillarity and Wetting Phenomena: Drops, Bubbles, Pearls, Waves, Springer, New York, 2004.

[19] P.G. de Gennes, On the fluid/wall slippage, Langmuir 8 (2002) 3413-3414, arXiv:0115383.

[20] P. Tabeling, Ed., Microfluidics, Comptes Rendus Physique, 5 (2004) 2-608.

[21] H. Gouin, S. Gavrilyuk, Dynamics of liquid nanofilms, Int. J. Eng. Sci. 46 (2008) 1195-1202, arXiv:0809.3489.

[22] B. Widom, What do we know that van der Waals did not know?, Physica A 263 (1999) 500-515.

[23] J.S. Rowlinson, B. Widom, Molecular Theory of Capillarity, Clarendon Press, Oxford, 1984.

[24] Y. Rocard, Thermodynamique, Masson, Paris, 1952.

[25] V.C. Weiss, Theoretical description of the adsorption and the wetting behavior of alkanes on water, J. Chem. Phys. 125 (2006) 084718.

[26] L.M. Pismen, Y. Pomeau, Disjoining potential and spreading of thin liquid layers in the diffuse-interface model coupled to hydrodynamics, Phys. Rev. E. 62 (2000) 2480-2492. 
[27] H. Gouin, Utilization of the second gradient theory in continuum mechanics to study the motion and thermodynamics of liquid-vapor interfaces, Physicochemical Hydrodynamics, B Physics 174 (1987) 667-682.

[28] H. Gouin, L. Espanet, Bubble number in a caviting flow, Comptes Rendus Acad. Sci. Paris IIb 328 (2000) 151-157, arXiv:0807.5023.

[29] Handbook of Chemistry and Physics, 65th Edition, CRC Press, Boca Raton, 1984-1985.

[30] D. Mattia, Y. Gogotsi, Review: static and dynamic behavior of liquids inside carbon nanotubes, Microfluid Nanofluid 5 (2008) 289-305.

[31] G.K. Batchelor, An introduction to fluid dynamics, Cambridge University Press, 1967.

[32] N.V. Chuarev, Thin liquid layers, Colloid J. 58 (1996) 681-693.

[33] T.D. Blake, Slip between a liquid and a solid - D.M. Tolstoi (1952) theory reconsidered, Colloids Surf. 47 (1990) 135-145.

[34] T.N. Wenzel, Surface roughness and contact angle, J. Phys. Colloid. Chem. 53 (1949) 1466-1467. 